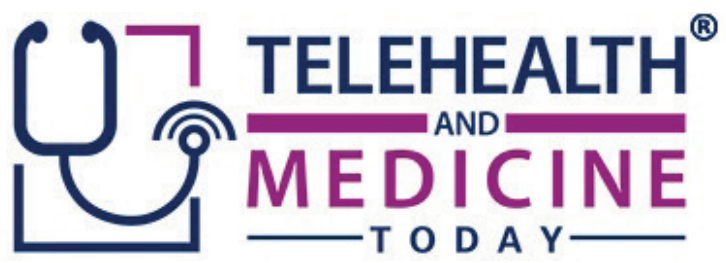

\title{
Emergency Medical Technician-Facilitated Telehealth Visits: A New Model to Expand Home-Based Primary Care for Homebound Seniors
}

Karen A. Abrashkin ${ }^{1}$, A. Camille McBride, ${ }^{1}$ Jill C. Slaboda, ${ }^{2}$ Michael Kurliand, ${ }^{2}$ Amparo Abel-Bey, ${ }^{1}$ Atika Turkistani, ${ }^{1}$ Kayla Finuf, ${ }^{1}$ Renee Pekmazaris ${ }^{1}$

Affiliations: ${ }^{1}$ Northwell Health, New Hyde Park, NY, USA; ${ }^{2}$ West Health, La Jolla, CA, USA

Corresponding Author: Karen A. Abrashkin, Email: kabrashkin@northwell.edu

Keywords: Emergency Medical Technician, Home-Based Primary Care, Homebound, Mobile Telemedicine Technician, Physician Extender, Telehealth, Telemedicine

Section: Proof of concept

Objective: As the number of older adults living in the United States grows, the gap between the capacity of home-based primary care (HBPC) services and the community demand will continue to widen. Older adults, living longer with mobility difficulties and multiple chronic medical conditions, often prefer to age in place, and new models of care are needed to meet this need. This article provides a framework for an innovative emergency medical technician (EMT)-facilitated telehealth program, the mobile telemedicine technician (MTT) program, which aims to increase access to medical care and efficiency within an HBPC program.

Design: A descriptive framework outlining the deployment of an innovative telehealth model.
Setting: An HBPC program serving homebound seniors in downstate New York.

Participants: Homebound individuals enrolled in an HBPC program with advanced age (over half $>90$ years), 67\% with 5-6 activities of daily living $(A D L)$ dependencies, and high rates of dementia, congestive heart failure, chronic obstructive pulmonary disease (COPD), and diabetes requiring evaluation and treatment of acute conditions.

Interventions: $H B P C$ program enrollees requiring evaluation and treatment of acute conditions received a home visit from a telehealthenabled EMT who has received additional training to provide in-home care. Following an evaluation, the EMT facilitated a telehealth visit 
via a two-way video conference between the patient and the primary care physician.

Main outcome measures: Description of a novel telehealth care model, preliminary results from the first 100 MTT visits including the reason for visit, patient/caregiver, physician, and telehealthenabled EMT satisfaction survey results.

Results: The primary care provider was able to evaluate twice as many patients in a given time period using the new model as in the regular home visit care model. The most common visit reasons were related to skin conditions (22\%), neurological conditions (19\%), cardiovascular conditions (16\%), and respiratory conditions (15\%). Satisfaction rates were high from patients/caregivers (45\% response rate, $60 \%$ strongly agreed and 29\% agreed that they were satisfied with the care delivery experience), physician (six surveys over time from one physician, 100\% strongly agreed on the effectiveness of care delivery model), and telehealth-enabled EMTs (eight surveys from four EMTs, 100\% strongly agreed that they were satisfied with the care delivery experience).

Conclusions: In this descriptive article, we outline a new model of care using telehealth-enabled EMTs making home visits to connect with a patient's primary care physician who is centrally located. This model shows promise for expanding primary care services within the home.

\section{INTRODUCTION}

While approximately 2 million adults aged 65 and older are currently considered homebound, ${ }^{1}$ the number of older adults living in the United States is expected to grow to more than $20 \%$ of the population by $2060,2,3$ likely increasing the number of homebound individuals substantially. Homebound adults aged 65 and older often have multiple chronic conditions and greater functional limitations than nonhomebound older adults. ${ }^{4}$ For these individuals, leaving the home for medical care is often difficult, and, when a medical issue arises, they may initially go without or delay care. This lack of primary care services can lead to an overreliance on emergency medical and hospital services. Evidence supports that older adults and homebound individuals use emergency medical services (EMS) at a disproportionately higher rate and are more likely to be hospitalized than younger adults and nonhomebound individuals, respectively. ${ }^{5}$

Home-based primary care (HBPC) is a medical model that transitions patient-centered care from outpatient clinics to the patient's home. HBPC has been shown to improve the quality of life and satisfaction with care for older adult patients and reduces the burden on their caregivers. ${ }^{6}$ Additionally, HBPC improves access to medical care while reducing hospitalizations and total cost of care. ${ }^{7} \mathrm{HBPC}$ uses an interdisciplinary team (IDT) that includes nurse, social worker, and physician or nurse practitioner to deliver care to patients with multiple chronic conditions such as dementia, congestive heart failure and/or diabetes, and functional status decline. HBPC allows older adults to age in place, which data show is preferred by the majority of older adults, and is beneficial for mental and physical health. Unfortunately, the need for HBPC services outstrips capacity. Analysis of 2013 Medicare fee-for-service program data showed that the majority of Americans lack access to HBPC by living more than 30 miles from a high-volume $(1,000$ or more visits) HBPC program. ${ }^{8}$ Further compounding this provider shortage is the high cost of operating an HBPC practice which is physician-intensive and relies on high-touch models of care. In order to grow programs to meet the needs of communities across the country, HBPC practices will need to explore innovative care models, and telehealth may be one such option. 
In this article, we describe and present preliminary data for an innovative emergency medical technician (EMT)-facilitated telehealth program, the mobile telemedicine technician (MTT) program, that was implemented in a downstate New York HBPC practice. The model builds on the practice's prior experience in telehealth deployment and aims to increase access to medical care and efficiency within the program by allowing twice the number of patient evaluations as would be possible using a traditional in-home visit structure. In addition to visit statistics, preliminary data regarding the satisfaction of patients/caregivers, physicians, and telehealthenabled EMTs are presented. This article aims to provide a framework for an EMT physician extender model and to inform the growing fields of both telehealth as well as HBPC.

\section{METHODS}

Our HBPC practice is located in downstate New York, and provides care to over 2,000 unique individuals, annually. Enrollees are homebound, generally older (average age 86 years), have multiple chronic conditions in an advanced state, multiple activity of daily living dependencies, and have had nonelective hospital utilization in the year prior to enrollment. The program utilizes geographically deployed teams of providers (physicians and nurse practitioners), nurses, social workers, and medical coordinators that provide routine and acute primary care visits in the home. A high-touch approach is needed to effectively care for homebound individuals with advanced illness, and routine in-home provider follow-up visits are scheduled every 2 to 4 months depending on a patient's level of clinical stability. All provider visits are augmented with telephonic and in-person nurse or social worker visits at regular intervals. In-home acute visits for changes in condition are scheduled on an as-needed basis by providers, nurses, and social workers.
By addressing both the medical and psychosocial components of disease, care teams aim to decrease disease burden, symptoms, stress on caregivers, and unwanted or potentially avoidable medical care. The program partners with a 24/7 telephonic nurse triage program, home care nursing services, infusion therapy, and hospice. Additionally, our health system is home to a large EMS agency with over 600 emergency responders (emergency medical technicians and paramedics), including a large community paramedicine program that partners closely with our HBPC program.

The MTT program utilizes telehealth-enabled EMTs to provide in-home evaluation of clinical changes in condition with decisional and clinical oversight by the patient's primary care physician via two-way, HIPAA compliant telehealth software and tablet technology (American Well, Boston, MA). EMTs from our health system's EMS agency with 3 or more years of experience and access to a personal vehicle were invited to apply for program participation. Four EMTs were selected and participated in a 2-day training including 1 day of didactics and 1 day of field observation. The didactic portion of the training included an introduction to program operations, population health, social determinants of health, motivational interviewing, and topics specific to the physical care of the homebound older adult patient including physical exam, an introduction to wound care, and advance care planning. Telehealth training included technical aspects of the platform used to facilitate the patient and the physician interaction as well as telehealth etiquette. The second day of EMT training was dedicated to field observation of HBPC physicians. The MTT program physician participated in training that included online telehealth training as well as in peer-to-peer shadowing and coaching from other telehealth providers in the organization. 
Visits were scheduled as outlined in Figure 1. EMTs, who were supplied with telehealth software enabled tablets, mobile Wi-Fi capabilities, tools for assessment of patient vitals and physical examination, staggered patient evaluation and telehealth visits with the distant site physician (physically located in the practice), thereby allowing for a physician to evaluate twice as many patients in a given time period as in a traditional home visit model. As this model was designed as a physician extender program, allowing the physician's clinical judgment to be applied across a larger population of patients than could be seen in a traditional model, physicians used clinical judgment to select patients appropriate for visits with an emphasis on unscheduled evaluation and treatment of acute conditions (e.g., a new rash or increased swelling) or follow-up after a care plan change (e.g., a blood pressure check after medication initiation). Most patients were selected for visits on the day of the EMT visit, with a maximum of 3 days prior to the EMT visit. The medical coordinator personnel provided route mapping to optimize travel efficiency for the EMTs who utilized their private vehicles. During the visit, the EMT performed a physical exam including vital signs, and could perform additional functions such as a home-safety evaluation, medication review, and assessment of social determinants of health, when indicated. The EMT then connected to the remote physician who is the patient's primary care physician and had access to medical records in real time. At the end of the visit, paper satisfaction surveys were left in the home for patients or caregivers to complete.

Primary care provider (PCP) documentation of the visit was completed in the ambulatory electronic health record and EMT documentation was completed in Research Electronic Data Capture (REDCap). EMT and physician satisfaction surveys were completed throughout the program at regular intervals were completed in REDCap. This project was evaluated by our institution's Institutional Review Board and deemed as quality improvement.

\begin{tabular}{|c|c|c|c|}
\hline Time & MTT 1 & Remote Primary Care Provider & MTT 2 \\
\hline $10-10: 30$ & $\begin{array}{c}\text { Evaluation \& } \\
\text { Video } \\
\text { Conference }\end{array}$ & Video Conference MTT 1 & Travel \\
\hline $10: 30-11$ & Travel & Video Conference MTT 2 & $\begin{array}{c}\text { Evaluation \& } \\
\text { Video } \\
\text { Conference }\end{array}$ \\
\hline $11-11: 30$ & $\begin{array}{c}\text { Evaluation \& } \\
\text { Video } \\
\text { Conference }\end{array}$ & Video Conference MTT 1 & Travel \\
\hline $11: 30-12$ & Travel & Video Conference MTT 2 & $\begin{array}{c}\text { Evaluation \& } \\
\text { Video } \\
\text { Conference }\end{array}$ \\
\hline $12-12: 30$ & $\begin{array}{c}\text { Evaluation \& } \\
\text { Video } \\
\text { Conference }\end{array}$ & Video Conference MTT 1 & Travel \\
\hline $12: 30-1$ & Travel & Video Conference MTT 2 & $\begin{array}{c}\text { Evaluation \& } \\
\text { Video } \\
\text { Conference }\end{array}$ \\
\hline $1-1: 30$ & & & 1 \\
\hline $1: 30-2$ & $\nabla$ & & $\nabla$ \\
\hline
\end{tabular}

Figure 1-Workflow illustrating staggered EMT travel and telehealth visits with the physician located in the practice. Through the MTT program, the remote PCP can evaluate and manage twice the number of patients as when making traditional home visits. 
Table 1. Patient characteristics for first 100 MTT visits $(n=78)$

\begin{tabular}{|c|c|}
\hline Characteristic & $n(\%)$ \\
\hline \multicolumn{2}{|l|}{ Sex } \\
\hline Male & $23(29)$ \\
\hline Female & $55(71)$ \\
\hline \multicolumn{2}{|l|}{ Age } \\
\hline$<70$ & $6(8)$ \\
\hline $70-79$ & $12(15)$ \\
\hline $80-89$ & $20(26)$ \\
\hline$\geq 90$ & $40(51)$ \\
\hline \multicolumn{2}{|l|}{ ADL dependencies* } \\
\hline 0 & $8(10)$ \\
\hline $1-2$ & $12(15)$ \\
\hline $3-4$ & $5(6)$ \\
\hline $5-6$ & $52(67)$ \\
\hline \multicolumn{2}{|l|}{ Chronic conditions } \\
\hline Dementia & $39(50)$ \\
\hline Pressure ulcers & $12(15)$ \\
\hline Diabetes mellitus & $23(29)$ \\
\hline Congestive heart failure & $24(31)$ \\
\hline Chronic obstructive pulmonary disease & $11(14)$ \\
\hline Protein-calorie malnutrition & $9(12)$ \\
\hline \multicolumn{2}{|l|}{ Insurance } \\
\hline Medicare primary & $32(41)$ \\
\hline Medicaid primary & $1(1)$ \\
\hline Private & $45(58)$ \\
\hline
\end{tabular}

*Activities of daily living included bathing, toileting, feeding, transferring, walking, and dressing.

\section{RESULTS}

The MTT program was implemented in July 2019 with the first 100 visits completed by September 2019. In each session, the physician was able to evaluate up to twice the number of patients as would be evaluated in the traditional model (10 vs. 5 per session). Patient characteristics for the first 100 visits (78 unique patients) are provided in Table 1. Our sample was mostly female (71\%), generally above 80 years old, and most needed assistance with 5-6 activities of daily living (ADL) (67\%). Most common chronic conditions of patients receiving visits included dementia (50\%), diabetes mellitus (29\%), and congestive heart failure $(31 \%)$.

The most common reasons for visits are presented in Table 2 and included integumentary (22\%, most commonly rashes), neurological (19\%, most commonly generalized weakness/ malaise), cardiovascular (16\%, most commonly fluid overload), and respiratory (15\%, most commonly cough). 
Table 2: Reasons for visits for first 100 MTT visits

\begin{tabular}{|c|c|c|c|}
\hline Category & $n$ & Subcategory & $n$ \\
\hline \multirow[t]{3}{*}{ Cardiovascular } & \multirow[t]{3}{*}{16} & Fluid overload & 7 \\
\hline & & Hypertension & 4 \\
\hline & & Hypotension & 5 \\
\hline \multirow[t]{4}{*}{ Gastrointestinal } & \multirow[t]{4}{*}{7} & Abdominal pain & 3 \\
\hline & & Constipation & 1 \\
\hline & & Diarrhea & 2 \\
\hline & & Dysphagia & 1 \\
\hline \multirow[t]{3}{*}{ Genitourinary } & \multirow[t]{3}{*}{4} & Dysuria & 1 \\
\hline & & Hematuria & 2 \\
\hline & & Other & 1 \\
\hline \multirow[t]{3}{*}{ Infectious } & \multirow[t]{3}{*}{4} & Cellulitis & 2 \\
\hline & & Fever & 1 \\
\hline & & Sore throat & 1 \\
\hline \multirow[t]{6}{*}{ Integumentary } & \multirow[t]{6}{*}{22} & Bleeding & 3 \\
\hline & & Blister & 1 \\
\hline & & Rash & 11 \\
\hline & & Skin tear & 2 \\
\hline & & Gangrene & 2 \\
\hline & & Infection & 3 \\
\hline \multirow[t]{2}{*}{ Musculoskeletal } & \multirow[t]{2}{*}{5} & Joint pain & 4 \\
\hline & & Back pain & 1 \\
\hline \multirow[t]{6}{*}{ Neurological } & \multirow[t]{6}{*}{19} & Agitation & 2 \\
\hline & & Altered mental status & 6 \\
\hline & & Dizziness & 2 \\
\hline & & Generalized weakness/malaise & 7 \\
\hline & & Generalized pain & 1 \\
\hline & & Stiffness/Parkinson's symptoms & 1 \\
\hline \multirow[t]{2}{*}{ Ophthalmologic } & \multirow[t]{2}{*}{3} & Pink eye & 2 \\
\hline & & Eye discharge & 1 \\
\hline \multirow[t]{3}{*}{ Other } & \multirow[b]{3}{*}{4} & Ear pain & 1 \\
\hline & & Lab abnormalities & 2 \\
\hline & & Medication question & 1 \\
\hline \multirow[t]{3}{*}{ Respiratory } & \multirow[t]{3}{*}{15} & Cough & 10 \\
\hline & & Shortness of breath & 3 \\
\hline & & Wheezing & 2 \\
\hline Rheumatologic & 1 & Gout & 1 \\
\hline Total & 100 & & 100 \\
\hline
\end{tabular}


Post-MTT visit outcomes included routine scheduled visit by the patient's primary care physician (61\%), scheduled phone call (16\%), visit by physician or nurse prior to a routinely scheduled visit (10\%), other (including hospice referral, home care referral, specialist visit, and repeat MTT visit in 1 week) (7\%), and EMS response for transport to the hospital (6\%). Emergency medical service transport to the hospital occurred when patients were clinically worse than anticipated at the time the MTT was scheduled: two patients needed urgent imaging for further evaluation, three patients had unstable vital signs, and one patient had altered mental status with suspected seizure.

Satisfaction rates were high from patients/ caregivers, the participating physician, and EMTs. Survey response rate for patients/ caregivers was $45 \%$ (35 completed by caregiver and 10 by patients) and $60 \%$ strongly agreed and $29 \%$ agreed that they were satisfied with the care delivery experience. The participating physician was surveyed each week for the first 4 weeks and then monthly. Of six surveys collected from the physician, $100 \%$ were answered as strongly agreed on effectiveness of the care delivery model. Surveys were collected monthly from four telehealth-enabled EMTs, with eight total surveys showing 100\% strongly agreed that they were satisfied with the care delivery experience.

\section{DISCUSSION}

Our study describes a framework and provides preliminary descriptive data for a unique model that uses EMTs to serve as physician extenders via telemedicine facilitation to expand in-home primary care services for older, chronically ill adults enrolled in an HBPC practice. Using this model, the physician was able to "see" double the number of patients as in a typical HBPC model. Our population of patients who, in the first 100 visits were mostly over 90 -years-old with 5-6 ADL dependencies and high rates of chronic conditions represent a growing demographic that has complex medical needs and prefers to receive care in the home. Preliminary data show that the most common reasons for MTT visits in this elderly population were related to rashes, assessment of fluid status, and cough. The MTT model shows promise in expanding access to HBPC while maintaining high levels of patient/caregiver, provider, and EMT satisfaction.

Most studies of EMTs delivering care via telehealth focus on EMS, with results showing potential for reduction in unnecessary emergency room transports in those receiving a video visit with an emergency room physician, ${ }^{9}$ enhanced diagnosis of acute myocardial infarction via electrocardiogram (EKG) transmission to a remote physician, stroke diagnosis via physician video evaluation, and disaster response. ${ }^{10,11}$ Utilization of EMTfacilitated telehealth in EMS responses for individuals with complaints deemed related to primary care showed success in preventing unnecessary emergency department (ED) transports, ${ }^{12}$ and experience in the Veteran's Association has provided extensive experience in varying telehealth use cases, including mental health services. ${ }^{13}$ Experiences, including our own direct-to-consumer pilot ${ }^{14}$ in our HBPC practice, have shown limited usability and uptake in the frail elderly population. ${ }^{15}$ Studies utilizing EMTs in a primary care environment have not been undertaken to date, to our knowledge.

With lessons learned from our first 100 visits, we will expand our care model from 1 to 2 days per week and expand the number of involved primary care physicians from one to four. This expansion will allow us to better refine 
operations, evaluate clinical and financial outcomes, and learn from patient/caregiver, physician, and EMT perceptions of the model. Early lessons learned have led to model changes, such as increased patient/caregiver education when anxiety over loss of in-person physician visits was detected. Operational lessons including scheduling and routing challenges have been valuable as well. Most fundamentally, whether a program such as this could allow for an increase in patient census and maintenance of clinical outcomes without an increase in physician staff is of paramount importance given the community need for in-home primary care services.

It is important to note that our MTT model and proposed evaluation of the program to include total cost of care evaluation will be limited by several factors. Lack of randomization will be the most significant limitation, as it is feasible that individuals selected for MTT visits were, regardless of the MTT visit, on a trajectory of illness different from those who did not receive visits. It is also feasible that individuals with certain characteristics, such as certain socioeconomic characteristics or medical literacy rates, are more likely to accept care via this novel delivery model.

HBPC physician extender programs such as the MTT model face a fundamental challenge: the innovation may be beneficial to patients and care delivery, but reimbursement mechanisms do not exist to sustain the work. At the time of our program's initiation, telehealth reimbursement through Medicare did not compensate for visits originating in the home in an urban environment, regardless of whether the patient was able to access traditional care settings. Changes in reimbursement that have taken place since the COVID-19 pandemic may provide additional revenue sources for such programs, as the home now, at least during the declared state of emergency, qualifies as an originating site for telehealth reimbursement. Additionally, new Capability Maturity Model Integration (CMMI) models, including direct contracting and emergency triage, treat, and transport (ET3), may allow for care model innovation of this sort, providing upfront funding for programs to manage complex care in the home and assuming some level of upside and downside risk for outcomes and patient total cost of care, and, in the case of ET3, reimbursing EMS agencies for care outside of transport to a hospital setting.

\section{CONCLUSION}

While our study of this new care model is observational in nature, we hope that our experiences and preliminary data will help inform local and national development of care delivery models, including payment and reimbursement, for HBPC practices and those aiming to serve high risk and high needs individuals in the home.

Acknowledgments: The authors would like to thank Northwell's Center for Emergency Medical Services for their invaluable partnership in the development of this new model of care, the House Calls physicians and all staff who have planned, coordinated, and conducted these telehealth visits, and their patients and families who have accepted this new model with openness and enthusiasm.

Conflicts of Interest: The authors declare no potential conflicts of interest. This manuscript is exclusively submitted to TMT, with the understanding that it has not been published elsewhere, in any form, and will not be submitted elsewhere. 
Contributors: K. Abrashkin, J. Slaboda, and M. Kurliand conceptualized the program, designed the study, and drafted the manuscript. A. McBride, A. Abel-Bey, and K. Finuf drafted the manuscript. A. Turkistani and R.

Pekmazaris critically revised the study. K. Abrashkin, J. Slaboda, M. Kurliand, and A. McBride conducted the data synthesis and interpretation. All authors approved the final draft of the manuscript submitted for publication.

Funding Statement: Funding for this study was provided by a grant from the Gary and Mary West Health Institute.

\section{REFERENCES}

1. Ornstein KA, Leff B, Covinsky KE, et al. Epidemiology of the homebound population in the United States. JAMA Intern Med. 2015;175(7):1180-6. https://doi. org/10.1001/jamainternmed.2015.1849

2. Mather M, Kilduff L, Scommegna P. Fact sheet: Aging in the United States [Internet]. Population Reference Bureau; 2019 [cited 2020 Apr 8]. Available from: https://www.prb.org/aging-unitedstatesfact-sheet/.

3. West LA, Cole S, Goodkind D, He W. 65 in the United States: 2010 special studies current population reports [Internet]. US Dept of Health and Human Services, US Dept of Commerce; 2014 [cited 2020 Apr 8]. Available from: https://www. census.gov/content/dam/Census/library/ publications/2014/demo/p23-212.pdf.

4. Soones T, Federman A, Leff B, Siu AL, Ornstein K. Two-year mortality in homebound older adults: An analysis of the National Health and Aging Trends Study. J Am Geriatr Soc. 2016;65(1):123-9. https://doi.org/10.1111/jgs.14467

5. Abrashkin KA, Poku A, Ramjit A, et al. Community paramedics treat high acuity conditions in the home: A prospective observational study. BMJ Support Palliat
Care. 2019. https://doi.org/10.1136/ bmjspcare-2018-001746

6. Stall N, Nowaczynski M, Sinha SK. Systematic review of outcomes from home-based primary care programs for homebound older adults. $J$ Am Geriatr Soc. 2014;62(12):2243-51. https://doi. org/10.1111/jgs. 13088

7. Edes T, Kinosian B, Vuckovic NH, Nichols LO, Becker MM, Hossain M. Better access, quality, and cost for clinically complex veterans with home-based primary care. J Am Geriatr Soc. 2014;62(10):1954-61. https://doi.org/10.1111/jgs.13030

8. Yao N, Ritchie C, Camacho F, Leff B. Geographic concentration of home-based medical care providers. Health Affairs. 2016;35(8):1404-9. https://doi.org/10.1377/ hlthaff.2015.1437

9. Langabeer JR, Champagne-Langabeer T, Alqusairi D, et al. Cost-benefit analysis of telehealth in pre-hospital care. $J$ Telemed Telecare. 2016;23(8):747-51. https://doi. org/10.1177/1357633X16680541

10. Majors-Foley K. Telemedicine in prehospital stroke evaluation and thrombolysis: Taking stroke treatment to the doorstep. J Emerg Med. 2016;51(1):93-4. https://doi.org/10.1016/j. jemermed.2016.06.024

11. Winburn AS, Brixey JJ, Langabeer J, Champagne-Langabeer T. A systematic review of prehospital telehealth utilization. $J$ Telemed Telecare. 2017;24(7):473-81. https://doi. org/10.1177/1357633X17713140

12. Champagne-Langabeer T, Langabeer JR, Roberts KE, et al. Telehealth impact on primary care related ambulance transports. Prehosp Emerg Care. 2019;23(5):712-7. https://doi.org/10.1080/10903127.2019.156 8650

13. Dang S, Olsan T, Karuza J, et al. Telehealth in home-based primary care: Factors and challenges associated with integration into veteran care. $J$ Am Geriatr Soc. 2019;67(9):1928-33. https://doi. org/10.1111/jgs.16045 
14. Latus-Olaifa O, Norman GJ, Kurliand M, et al. Not yet ready for prime time: Video visits in a home-based primary care program. $J \mathrm{Am}$ Geriatr Soc. 2019;67(10):2202-4. https://doi. org/10.1111/jgs.16064

15. De Cola MCD, Maresca G, D’Aleo G, et al. Teleassistance for frail elderly people: A usability and customer satisfaction study. Geriatr Nurs. 2020. https://doi. org/10.1016/j.gerinurse.2020.01.019
Copyright Ownership: This is an open access article distributed in accordance with the Creative Commons Attribution Non Commercial (CC BY-NC 4.0) license, which permits others to distribute, adapt, enhance this work non-commercially, and license their derivative works on different terms, provided the original work is properly cited and the use is non-commercial. See: http:// creativecommons.org/licenses/by-nc/4.0. 